Increasing existing $\beta$ cell mass is a promising avenue for the treatment of type 2 diabetes, and initial positive results have been obtained with one such agent, exendin 4, an analog of glucagon-like peptide 1 (8-10). Likewise, the new model will allow researchers to uncover negative effects of commonly used drugs in $\beta$ cell regeneration. Finally, these mice will also be instrumental in elucidating the molecular mechanisms that govern the proliferative response of the mature $\beta$ cell.

Address correspondence to: Klaus H. Kaestner, Department of Genetics and Institute for Diabetes, Obesity, and Metabolism,
560A CRB/6140, University of Pennsylvania, Philadelphia, Pennsylvania 19104, USA. Phone: (215) 898-8759; Fax: (215) 573-5892; E-mail: kaestner@mail.med.upenn.edu.

1. Shapiro, A.M., et al. 2000. Islet transplantation in seven patients with type 1 diabetes mellitus using a glucocorticoid-free immunosuppressive regimen. N. Engl. J. Med. 343:230-238.

2. Bonner-Weir, S., and Weir, G.C. 2005. New sources of pancreatic beta-cells. Nat. Biotechnol. 23:857-861.

3. Dor, Y., Brown, J., Martinez, O.I., and Melton, D.A. 2004. Adult pancreatic beta-cells are formed by self-duplication rather than stem-cell differentiation. Nature. 429:41-46.

4. Teta, M., Rankin, M.M., Long, S.Y., Stein, G.M., and Kushner, J.A. 2007. Growth and regeneration of adult beta cells does not involve specialized progenitors. Dev. Cell. 12:817-826.

5. Nir, T., Melton, D.A., and Dor, Y. 2007. Recovery from diabetes in mice by $\beta$ cell regeneration. J. Clin. Invest. 117:2553-2561. doi:10.1175/JCI32959.

6. Brownlee, M. 2003. A radical explanation for glucose-induced $\beta$ cell dysfunction. J. Clin. Invest. 112:1788-1790. doi:10.1172/JCI200320501.

7. Wajchenberg, B.L. 2007. beta-cell failure in diabetes and preservation by clinical treatment. Endocr. Rev. 28:187-218.

8. Brubaker, P.L., and Drucker, D.J. 2004. Minireview: Glucagon-like peptides regulate cell proliferation and apoptosis in the pancreas, gut, and central nervous system. Endocrinology. 145:2653-2659.

9. DeFronzo, R.A., et al. 2005. Effects of exenatide (exendin-4) on glycemic control and weight over 30 weeks in metformin-treated patients with type 2 diabetes. Diabetes Care. 28:1092-1100.

10. Kendall, D.M., et al. 2005. Effects of exenatide (exendin-4) on glycemic control over 30 weeks in patients with type 2 diabetes treated with metformin and a sulfonylurea. Diabetes Care. 28:1083-1091.

\title{
Deepening our understanding of immune sentinels in the skin
}

\author{
Frank O. Nestle ${ }^{1}$ and Brian J. Nickoloff ${ }^{2}$
}

${ }^{1}$ St. John's Institute of Dermatology, Division of Genetics and Molecular Medicine, King's College London School of Medicine, London, United Kingdom. 2Department of Pathology and Department of Microbiology and Immunology, Oncology Institute, Cardinal Bernardin Cancer Center,

Loyola University, Chicago, Illinois, USA.

\begin{abstract}
Advances in our understanding of the skin immune system have a major impact on studies of skin autoimmunity, graft-versus-host disease, inflammation, and cancer as well as on the development of novel vaccines and immunotherapy approaches. In this issue of the JCI, Zaba et al. carefully dissected the complex network of DCs and macrophages residing in normal human skin and defined novel phenotypic markers for these immunocytes (see the related article beginning on page 2517). These studies provide the basis for better insight into the role of important immune sentinels contributing to the maintenance of skin tissue homeostasis and lay the foundation for future studies of the skin immune system.
\end{abstract}

The skin is one of the largest organs of the body and has a variety of different functions including providing the first line of defense against invading pathogens. Upon casual inspection, normal human skin does not appear to be a major reservoir of immune sentinels and effector cells. However, the absence of inflammation belies the significant number and phenotypic complexity of tissue-resident T cells, macrophages, and DCs that are becoming more widely appreciated by immunolo-

Nonstandard abbreviations used: BDCA, blood DC antigen; DC-SIGN, DC-specific ICAM-grabbing nonintegrin; DDC, dermal DC; FXIIIa, factor XIIIa; LC, Langerhans cell; PDC, plasmacytoid pre-DC.

Conflict of interest: The authors have declared that no conflict of interest exists.

Citation for this article: J. Clin. Invest. 117:2382-2385 (2007). doi:10.1172/JCI33349. gists interested in immune surveillance, autoimmunity, chronic inflammatory diseases in the skin, and beyond (1). Once the primary barrier function of skin (e.g., disruption of the outermost dead layer of cells forming the stratum corneum) is breached, immune sentinel and effector cells are poised to provide rapid and efficient immunological backup to restore tissue homeostasis. For example, it is not widely appreciated that there are approximately $2 \times 10^{10}$ skin-resident $\mathrm{T}$ cells, i.e., twice the total number of $\mathrm{T}$ cells in the circulation (2). A frequent misconception regarding cutaneous immunity is that "skin" equals "epidermis" (3). Once one probes beneath the basement membrane zone, there is a multitude of cells, including $\mathrm{T}$ lymphocytes, DCs, and macrophages, that are regarded as components of the dermal immune system and complement the overlying epidermal components to form the skin immune system. The dermal immune system provides a highly reactive immunologically based compartment that is crucially involved in the majority of chronic inflammatory skin disorders including psoriasis and atopic dermatitis (4). Here, we primarily focus on the dermal-based immune system under normal or noninflammatory skin conditions.

\section{Skin-resident sentinels of the immune system}

Skin-resident sentinels of the immune system include a spectrum of cells, ranging from motile DCs highly specialized in sensing danger and presenting antigens to tissue-resident macrophages specializing in phagocytosis, thereby clearing potentially dangerous substances and mediating tissue remodeling. Langerhans cells (LCs) are dendritic, antigen-presenting sentinels of the epidermis. LCs renew locally in epidermis and only recruit blood-borne precursors following injury (5). Even though LCs were described 140 years ago, their in vivo function still remains elusive. Recent scientific evidence obtained using LCdepleted genetically modified mice adds 


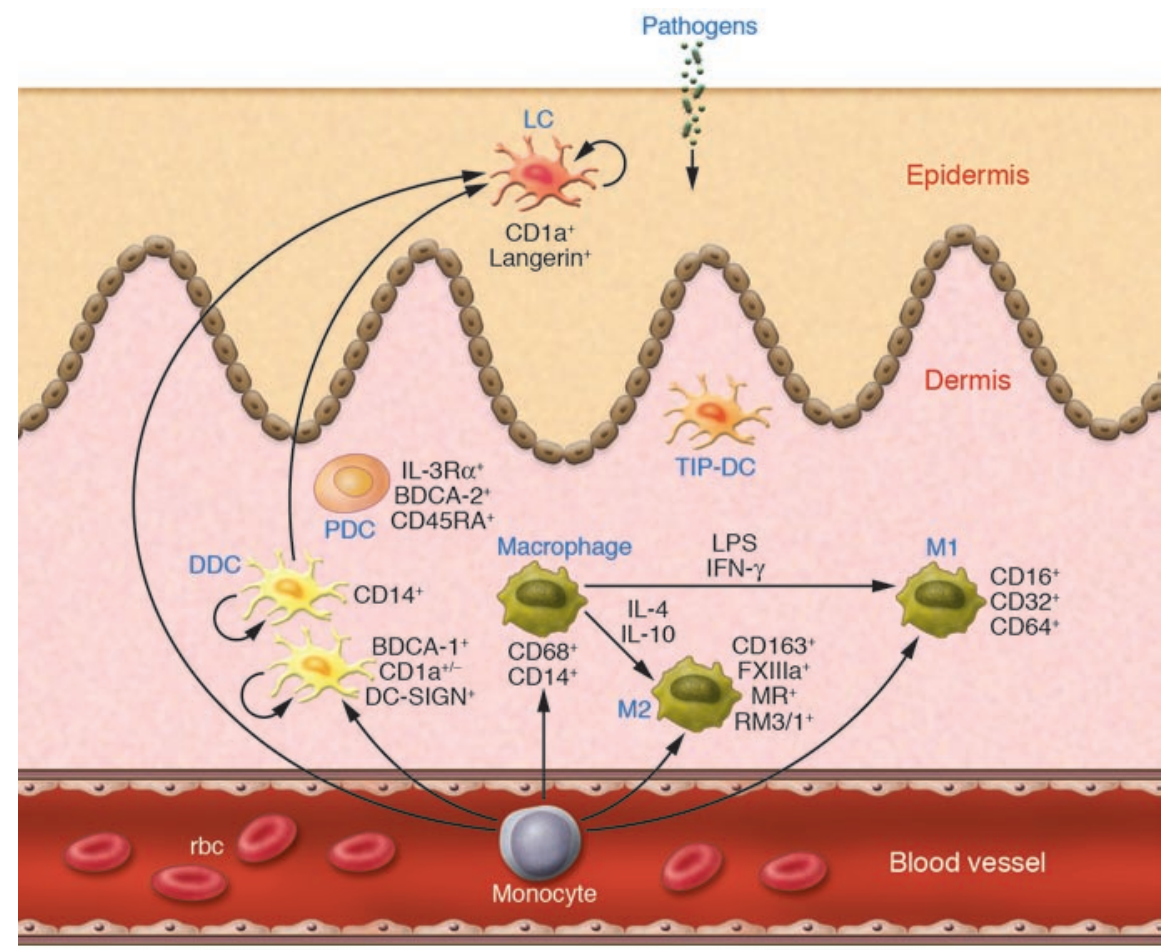

Figure 1

Normal human skin is characterized by an impressive diversity of immune sentinels. Skin-based DCs and macrophages sense invading pathogens and serve as sentinels, thereby alerting effectors of the innate and adaptive immune systems to potential danger to the host. Subsets of immune sentinels include CD1a+Langerin+ LCs located in the epidermis and various subtypes of DCs and macrophages in the dermis. In this issue of the $\mathrm{JCl}$, Zaba et al. (17) used mixed leukocyte assay to identify BDCA-1+ DDCs as the major immunostimulatory population in normal human skin. In addition, they found that the mononuclear cells expressing CD163 were less immunostimulatory, but possessed greater phagocytic activity and morphological features of macrophages. These recent morphological, immunophenotypic, and functional findings complement previous studies that defined additional mononuclear subsets including: PDCs, which are relatively rare in normal skin but are capable of producing type I IFNs and express BDCA-2, IL-3 receptor $\alpha$ (IL-3R $\alpha$ ), and CD45RA; CD14+ DDCs, which may develop into LCs under the influence of TGF- $\beta$; TNF- and iNOS-producing DCs (TIP-DC), characterized by their production of TNF- $\alpha$ and iNOS; and various macrophage subsets. Currently the macrophage population expressing CD68 and CD14 can be further subdivided into classically activated macrophages (M1), developing under the influence of LPS and IFN- $\gamma$, and alternatively activated macrophages (M2), developing under the influence of IL-4 and IL-10. The M1 type macrophage expresses CD16, CD32, and CD64, whereas the M2 type macrophage expresses CD163, FXIIIa, MR, and the marker RM3/1 as previously described (15). Circular arrows indicate the self-renewing potential of LCs and DDCs under conditions of tissue homeostasis. Moreover, circulating blood-derived monocytes are potential precursors of LCs, DDCs, and macrophages, especially under inflammatory conditions.

to potential confusion by demonstrating both immunoactivating and immunosuppressive roles of LCs (3). While LCs are wellstudied components of the skin immune system, dermal DCs (DDCs) were only identified and functionally characterized 14 years ago $(6,7)$. Despite the presence of significant numbers of DDCs in normal and pathological skin, the complexity of this cell type is just beginning to be understood. DDCs are defined by their characteristic phenotypic and functional properties including typical dendritic morphology, nonadherence to plastic, expression of high levels of adhesion and costimulatory molecules, and potent stimulatory capacity of naive and memory $T$ cells in the context of alloantigens and nominal antigens (8). They have potential to divide locally and thus maintain their tissue density in the steady state (9). A key unifying feature of DDCs is expression of the nonpolymorphic MHC-like lipid antigen-presenting molecule blood DC antigen-1 (BDCA-1; also known as CD1c) as well as C-type lectins such as the pathogen sensor DC-specific ICAM-grabbing nonintegrin (DC-SIGN; also known as CD209) and DEC205 (also known as CD205).

Three major DDC subpopulations have been characterized in the past: BDCA$1^{+} \mathrm{CD} 1 \mathrm{a}^{+} \mathrm{CD} 14^{-}$and BDCA- $1^{+} \mathrm{CD} 1 \mathrm{a}^{-} \mathrm{CD} 14^{-}$ DDCs have potent antigen-presenting capacity $(6,10)$, and BDCA $-1^{+} \mathrm{CD} 1 \mathrm{a}^{-} \mathrm{CD} 14^{+}$ DDCs have low antigen-presenting capacity but the potential to develop into CD $1 \mathrm{a}^{+}$ and Langerin-positive LCs under control of TGF- $\beta$ (11). There is also an important subset of proinflammatory DDCs (termed "TNF- and iNOS-producing DCs" in reference to their proinflammatory mouse DC counterparts; ref. 12). A recent addition to the family of skin immune sentinels is type I IFN-producing plasmacytoid pre-DCs (PDCs). These cells are rare in normal skin, but accumulate under conditions of chronic skin inflammation such as psoriasis and lupus erythematosus and have important functions in skin immunopathology $(13,14)$. A further component of the dermal immune system is the dermal macrophage. Although detailed functional studies are lacking, several surface markers including CD14, CD68, HLA-DR, RM3/1, and $\mathrm{CD} 11 \mathrm{c}$ have been used in the past to describe the phenotypic diversity of the dermal macrophage population $(15,16)$. 


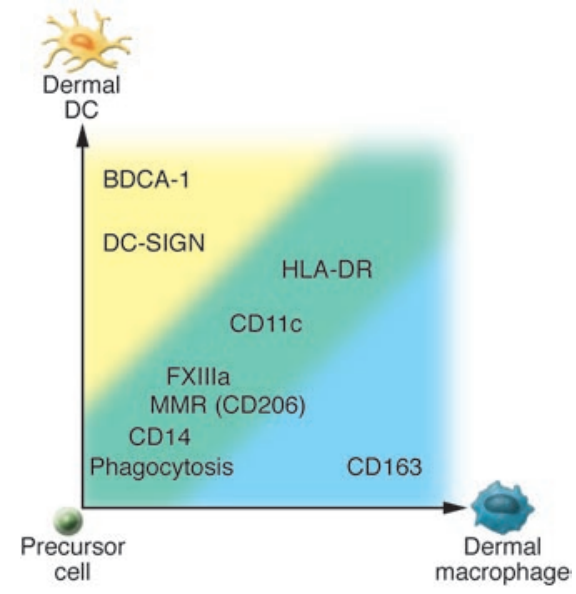

Figure 2

The spectrum of immune sentinels, portrayed in 2 dimensions, reflecting the heterogeneity and flexible functionality of the mononuclear subsets located in normal human dermis. At one end of the spectrum are DDCs expressing BDCA-1 (also known as CD1c) and DCSIGN (also known as CD209); at the other end are macrophages expressing CD163. Depending on the skin microenvironment and cellular activation state of the mononuclear cells, additional markers and flexible functionality may emerge between these ends of the spectrum, as reflected by expression of $\mathrm{MHC}$ class II antigen (i.e., HLA-DR), CD11c, FXIIIa, MMR (CD206), CD14, and varying degrees of phagocytic activity. At present, the physiological and pathological relevance for the recruitment of and phenotypic as well as functional interactions among these mononuclear cell subsets, not to mention the effector arms of the innate and adaptive immune system in the skin, is not completely understood.

\section{Diversity of DCs and macrophage subsets in the dermis}

In a study reported in this issue of the JCI, Zaba et al. (17) shed new light on the phenotype and function of DDCs and macrophages. Using a panel of monoclonal antibodies to carefully dissect the phenotypic expression profile of DDCs and macrophages, they found 2 major subpopulations of dermal immune sentinels. An overview of the mononuclear cell subsets located in the epidermis and dermis of normal human skin incorporating the findings of $\mathrm{Zaba}$ et al. is portrayed in Figure 1. The BDCA- $1^{+} \mathrm{CD} 11 \mathrm{c}^{+} \mathrm{HLA}-\mathrm{DR}^{+}$ DDCs possessed strong stimulatory capacity for allogeneic $\mathrm{T}$ cells, and a second macrophage-like population expressed CD163 and factor XIIIa (FXIIIa). CD163 is a hemoglobin/haptoglobin complex-binding macrophage scavenger receptor expressed on the majority of tissue macrophages (18) and can be readily added to the phenotypic criteria for skin macrophages. FXIIIa, best known for its role in coagulation, is also a tissue transglutaminase with a potential functional role in wound healing and other cutaneous disorders (4). Expression of FXIIIa is related to cell activation and inducible via IL-4 in alternatively activated macrophages (19). FXIIIa is also expressed in migratory DDCs $(6,11)$, monocyte-derived DCs $(20)$, hematopoietic progenitor cell-derived DDC equivalents (21), and a population of $\mathrm{MHC}$ class $\mathrm{II}^{+}$dendritic-appearing cells of the dermis termed "dermal dendrocytes" (22). Despite these new findings, an immediate question arises: What is the biological significance of FXIIIa expression by dermal macrophages and DCs? Rather than being a lineage-specific marker, accumulating data suggest that FXIIIa expression indicates an alternative cellular activation state induced by exposure of DCs and macrophages to IL-4 $(19,20)$. Furthermore, FXIIIa expression might indicate a potential functional role in tissue remodeling. Thus, the original description of FXIIIa dermal dendrocytes in various pathological states (22) might have included both DCs and macrophages with a dendritic morphology residing in a distinctive cytokine-containing (e.g., IL-4rich) skin microenvironment.

Accumulating data seem to indicate that there exists a continuum of phenotypes and functions and remarkable plasticity between tissue-resident DCs and macrophages (23). Based on the findings by Zaba et al. (17), it is clear that certain phenotypic markers may be useful to define mononuclear cell subsets at each end of a spectrum. For example, BDCA-1 $1^{+}$ cells identify DDCs with potent allostimulating properties, while the $\mathrm{CD} 163^{+}$ cells lacked significant allostimulatory capacity but possessed greater phagocytic activity. As portrayed in Figure 2, between these ends of the spectrum, there are dermal mononuclear cell subsets that express various markers that may identify cell types with more flexible functionality as regards antigen presentation and phagocytosis. Thus, it is now well established that immature DCs, including DDCs, can be phagocytic, a cellular function usually attributed to macrophages $(24,25)$. On the other hand, macrophages might be potent antigen-presenting cells for $\mathrm{CD}^{+}$ T cells (26). Expression of CD1a on DDCs indicates potent antigen-presenting function, while expression of CD14 indicates a precursor role for LCs (11). Thus, tissueresident mononuclear sentinels of the dermis are likely to exist in a pluripotent state and, depending on microenvironmental factors, may acquire an antigen-presenting mode, migratory mode, or tissue-resident phagocytic mode.

\section{Future directions and conclusions}

The present study by Zaba et al. (17) contributes to a deeper understanding of the complex world of DCs and macrophages residing in normal human skin. The study also raises important questions for future study. What is the role of human skin DCs versus macrophages in the induction of peripheral $T$ cell unresponsiveness under steady-state conditions (27)? What is the in vivo functional role of human skin DCs in priming and cross-priming of a pathogen-specific immune response, and can these properties be exploited for vaccination purposes? And finally, what role are these mononuclear cells playing in conditions of chronic inflammation and cancer in the skin? Despite these uncertainties, we now have better surface markers to delineate the confederacy of sentinel cells in skin, which will hopefully allow us to probe more deeply into proinflammatory, antiinflammatory, immunogenic, and tolerogenic DC and macrophage subsets and their potential roles in skin inflammation and skin cancer.

\section{Acknowledgments}

The authors thank Lori Kmet and Lynn Walter for manuscript preparation and acknowledge support from the NIH (grants AR40065 and CA39542), the Wellcome Trust, and the United Kingdom Department of Health through the NIH Research Biomedical Research Centre award to Guy's \& St. Thomas' National Health Service Foundation Trust in partnership with King's College London.

Address correspondence to: Brian J. Nickoloff, Loyola University Medical Center, 2160 S. First Avenue, Building 112 Room 301, Maywood, Illinois 60513, USA. Phone: (708) 327-3241; Fax: (708) 327-3239; E-mail: bnickol@lumc.edu. Or to: Frank O. Nestle, Division of Genetics and Molecular Medicine, King's College London School of Medicine, 8th Floor Guy's Tower, Guy's Hospital, London SE1 9RT, United Kingdom. Phone: 44-20-7188-9038; Fax: 44-207188-2585; E-mail: frank.nestle@kcl.ac.uk. 
1. Boyman, O., Conrad, C., Tonel, G., Gilliet, M., and Nestle, F.O. 2007. The pathogenic role of tissueresident immune cells in psoriasis. Trends Immunol. 28:51-57.

2. Clark, R.A., et al. 2006. The vast majority of CLA+ $\mathrm{T}$ cells are resident in normal skin. J. Immunol. 176:4431-4439.

3. Romani, N., et al. 2006. Epidermal Langerhans cells--changing views on their function in vivo. Immunol. Lett. 106:119-125.

4. Nickoloff, B.J. 1993. Dermal immune system. CRC Press. Boca Raton, Florida, USA. 340 pp.

5. Merad, M., et al. 2002. Langerhans cells renew in the skin throughout life under steady-state conditions. Nat. Immunol. 3:1135-1141.

6. Nestle, F.O., Zheng, X.G., Thompson, C.B., Turka, L.A., and Nickoloff, B.J. 1993. Characterization of dermal dendritic cells obtained from normal human skin reveals phenotypic and functionally distinctive subsets. J. Immunol. 151:6535-6545.

7. Lenz, A., Heine, M., Schuler, G., and Romani, N 1993. Human and murine dermis contain dendritic cells. Isolation by means of a novel method and phenotypical and functional characterization. J. Clin. Invest. 92:2587-2596.

8. Nestle, F.O., Filgueira, L., Nickoloff, B.J., and Burg, G. 1998. Human dermal dendritic cells process and present soluble protein antigens. J. Invest. Dermatol. 110:762-766

9. Bogunovic, M., et al. 2006. Identification of a radio-resistant and cycling dermal dendritic cell population in mice and men. J. Exp. Med. 203:2627-2638.

10. Angel, C.E., et al. 2006. Cutting edge: CD1a+ antigen-presenting cells in human dermis respond rap- idly to CCR7 ligands. J. Immunol. 176:5730-5734.

11. Larregina, A.T., et al. 2001. Dermal-resident CD14+ cells differentiate into Langerhans cells. Nat. Immunol. 2:1151-1158.

12. Lowes, M.A., et al. 2005. Increase in TNF-alpha and inducible nitric oxide synthase-expressing dendritic cells in psoriasis and reduction with efalizumab (anti-CD11a). Proc. Natl. Acad. Sci. U. S. A. 102:19057-19062.

13. Nestle, F.O., et al. 2005. Plasmacytoid predendritic cells initiate psoriasis through interferon-alpha production. J. Exp. Med. 202:135-143.

14. Wollenberg, A., et al. 2002. Plasmacytoid dendritic cells: a new cutaneous dendritic cell subset with distinct role in inflammatory skin diseases. J. Invest. Dermatol. 119:1096-1102.

15. Djemadji-Oudjiel, N., Goerdt, S., Kodelja, V., Schmuth, M., and Orfanos, C.E. 1996. Immunohistochemical identification of type II alternatively activated dendritic macrophages (RM 3/1+3, MS 1+/-, 25F9-) in psoriatic dermis. Arch. Dermatol. Res. 288:757-764

16. Weber-Matthiesen, K., and Sterry, W. 1990. Organization of the monocyte/macrophage system of normal human skin. J. Invest. Dermatol. 95:83-89.

17. Zaba, L.C., Fuentes-Duculan, J., Steinman, R.M., Krueger, J.G., and Lowes, M.A. 2007. Normal human dermis contains distinct populations of CD $11 \mathrm{c}^{+} \mathrm{BDCA}^{-1^{+}}$dendritic cells and $\mathrm{CD} 163^{+} \mathrm{FXIIIA}{ }^{+}$ macrophages. J. Clin. Invest. 117:2517-2525. doi:10.1172/JCI32282.

18. Fabriek, B.O., Dijkstra, C.D., and van den Berg, T.K. 2005. The macrophage scavenger receptor CD163. Immunobiology. 210:153-160.

19. Torocsik, D., Bardos, H., Nagy, L., and Adany, R.
2005. Identification of factor XIII-A as a marker of alternative macrophage activation. Cell. Mol. Life Sci. 62:2132-2139.

20. Grassi, F., et al. 1998. Monocyte-derived dendritic cells have a phenotype comparable to that of dermal dendritic cells and display ultrastructural granules distinct from Birbeck granules. J. Lenkoc. Biol. 64:484-493.

21. Caux, C., et al. 1996. CD34+ hematopoietic progenitors from human cord blood differentiate along two independent dendritic cell pathways in response to GM-CSF+TNF alpha. J. Exp. Med. 184:695-706.

22. Cerio, R., Griffiths, C.E., Cooper, K.D., Nickoloff, B.J., and Headington, J.T. 1989. Characterization of factor XIIIa positive dermal dendritic cells in normal and inflamed skin. Br.J. Dermatol. 121:421-431.

23. Gordon, S., and Taylor, P.R. 2005. Monocyte and macrophage heterogeneity. Nat. Rev. Immunol. 5:953-964.

24. Blander, J.M., and Medzhitov, R. 2006. On regulation of phagosome maturation and antigen presentation. Nat. Immunol. 7:1029-1035.

25. Filgueira, L., Nestle, F.O., Rittig, M., Joller, H.I., and Groscurth, P. 1996. Human dendritic cells phagocytose and process Borrelia burgdorferi. J. Immunol. 157:2998-3005.

26. Pozzi, L.A., Maciaszek, J.W., and Rock, K.L. 2005. Both dendritic cells and macrophages can stimulate naive $\mathrm{CD} 8 \mathrm{~T}$ cells in vivo to proliferate, develop effector function, and differentiate into memory cells. J. Immunol. 175:2071-2081.

27. Steinman, R.M., Hawiger, D., and Nussenzweig, M.C. 2003. Tolerogenic dendritic cells. Annu. Rev. Immunol. 21:685-711.

\title{
Taking aim at translation for tumor therapy
}

\author{
Bryan C. Barnhart ${ }^{1}$ and M. Celeste Simon'1,2,3,4
}

${ }^{1}$ Abramson Family Cancer Research Institute, University of Pennsylvania Cancer Center, ${ }^{2}$ Howard Hughes Medical Institute, and ${ }^{3}$ Department of Cancer Biology and ${ }^{4}$ Department of Cell and Developmental Biology, School of Medicine, University of Pennsylvania, Philadelphia, Pennsylvania, USA.

\begin{abstract}
Increased cap-dependent mRNA translation rates are frequently observed in human cancers. Mechanistically, many human tumors often overexpress the cap binding protein eukaryotic translation initiation factor 4E (eIF4E), leading to enhanced translation of numerous tumor-promoting genes. In this issue of the JCI, Graff and colleagues describe potent antitumor effects using second-generation antisense oligonucleotides for $\mathrm{EIF} 4 \mathrm{E}$ (see the related article beginning on page 2638). If their results are recapitulated in a clinical setting, this strategy will provide a promising antitumor therapy with broad-reaching applications.
\end{abstract}

Protein synthesis is required for many critical cellular processes, and cells regulate mRNA translation rates according to their needs. Interestingly, dysregulated translation has now been

Nonstandard abbreviations used: ASO, antisense oligonucleotide; 4E-BP, eIF4E binding protein; eIF4E, eukaryotic translation initiation factor 4E; mTORC1, mammalian target of rapamycin complex 1 .

Conflict of interest: The authors have declared that no conflict of interest exists.

Citation for this article: J. Clin. Invest. 117:2385-2388 (2007). doi:10.1172/JCI33107. linked to multiple human cancers $(1,2)$. Increased translation rates lead to an overproduction of proteins involved in proliferation, survival, metastasis, and other malignant characteristics (3-5). Protein synthesis regulation is complex, and its alteration in tumor cells occurs at numerous points. Many tumor-promoting mechanisms ultimately cause the activation of a critical regulator of capdependent translation, the eukaryotic translation initiation factor 4F (eIF4F) complex. Numerous human tumors exhibit inappropriate eIF4F activation, including lymphomas and breast, prostate, colorectal, head and neck, cervical, bladder, and lung cancers (3). Therefore, therapeutically targeting eIF4F activity is exceedingly attractive, as it would potentially be applicable to a broad range of human cancers. In this issue of the JCI, Graff and colleagues (6) report such a strategy, attacking one of the important components of eIF4F, eIF4E, with striking efficacy in tumor models. If this treatment is successful in the clinic, it holds great promise for use against many human tumors and may be especially effective if used in combination with more traditional chemotherapeutic treatments.

\section{Cap-dependent protein synthesis and its regulation}

To provide better insight into the underlying mechanism for this therapy and why it might ultimately be so effective against 